\title{
Dynamical Analysis and Control of a Chaotic Microelectromechanical Resonator Model
}

\author{
Dailhane G. Bassinello $\mathbb{D},{ }^{1}$ Angelo M. Tusset $\mathbb{D},^{2}$ \\ Rodrigo T. Rocha ${ }^{(D)}{ }^{2}$ and Jose M. Balthazar $\mathbb{I D}^{1,2}$ \\ ${ }^{1}$ Department of Electrical Engineering, São Paulo State University, 17033-360 Bauru, SP, Brazil \\ ${ }^{2}$ Federal University of Technology-Paraná, 84016-210 Ponta Grossa, PR, Brazil
}

Correspondence should be addressed to Rodrigo T. Rocha; digao.rocha@gmail.com

Received 26 January 2018; Accepted 10 April 2018; Published 15 May 2018

Academic Editor: Onome E. Scott-Emuakpor

Copyright (c) 2018 Dailhane G. Bassinello et al. This is an open access article distributed under the Creative Commons Attribution License, which permits unrestricted use, distribution, and reproduction in any medium, provided the original work is properly cited.

\begin{abstract}
The dynamic analysis and control of a nonlinear MEM resonator system are considered. Phase diagram, bifurcation diagram, and the $0-1$ test are applied to the analysis of the influence of the parameters on the dynamics of the system, whose parameters are damping coefficient, polarization of the voltage, and nonlinear stiffness term. The bifurcation diagram is used to demonstrate the existence of the pull-in effect. Numerical results showed that the parameters, which were taken into account, were significant, indicating that the response can be either chaotic or periodic behavior. In order to bring the system from a chaotic state to a periodic orbit, two controls are considered: the time-delayed feedback control and the sliding mode control.
\end{abstract}

\section{Introduction}

Microelectromechanical resonators were proposed in the middle of 1990s as a viable alternative for comb-drive actuators and other large conventional filters. Due to the possibility of microelectromechanical system (MEMS) applications, many researchers have dedicated their researches directed to MEMS. In [1] the nonlinear dynamics of micromechanical oscillators are explored experimentally, and a model was built to explain why high-order entrainment is seen only in doubly supported beams, and by its analysis it suggests that the strong amplitude-frequency relationship in doubly supported beams enables hysteresis, wide regions of primary entrainment, and high orders of sub- and superharmonic entrainment. In [2] a model for oscillations from the continuum description of the temperature and displacement field is considered. A bifurcation analysis of the model was performed, allowing estimating the threshold power for self-oscillation as a function of geometric and optical constants of the beam. In [3] the Mathieu-van der Pol Duffing equation is considered for presenting a MEMS model, and the authors investigated the dynamics of the system considering it forced by parametric and nonparametric excitations. In [4] a thermomechanical model of the system was developed, and its predictions were explored to explain and predict the entrainment phenomenon. The model is validated with experimental results and could also be used to analyze MEMS limit-cycle oscillators designed to achieve specific performance objectives.

In microelectromechanical systems (MEMS), nonlinearities may arise from a variety of sources, such as spring and damping mechanisms and resistive, inductive, and capacitive circuit elements. An interesting example of nonlinearity in MEMS was demonstrated in [5-8] where it is possible to observe that the nonlinear electrostatic force depends on the gap size between the two electrodes.

Due to the nonlinearities presented in the MEMS, chaotic motion may appear. Therefore, some methods may be applied to identify such irregular motion. One of the most effective methods is the 0-1 test that can be used for any system to identify chaotic dynamics. This test uses the temporal series data to characterize the dynamics of the system, analyzing its spectral and statistical properties based on the asymptotic properties of a Brownian motion [9-14]. 
The ability of the time-delayed feedback control in bringing a system with chaotic behavior to a periodic attractor system has attracted the attention of several researchers, both for numerical and experimental applications.

In [15], experimental results of the application of the time-delayed feedback control in a nonlinear oscillator are presented. The experimental system was an externally driven nonlinear oscillator, including a tunnel diode, as a negative resistance device. To electronically realize the control a special analogue circuit was designed, and the signal of the control is applied in the tunnel diode. The experimental results demonstrated the efficiency of the control.

In [16], the time-delayed feedback control is used in an Atomic Force Microscope microcantilever with chaotic behavior. The control of the chaotic behavior of the system was implemented by using the time-delayed feedback control. Furthermore, in [17], the time-delayed feedback control was used in the control of the chaotic behavior of an Atomic Force Microscope microcantilever. In both cases, the authors used a nonlinear mathematical model for the representation of the AFM, and the control was performed by means of numerical simulations. Those results showed the feasibility of the timedelayed feedback control for chaos control in the considered AFM system.

In addition, the time-delayed feedback control has been used in chaos control of smart systems composed of a pendulum coupled with shape memory alloy (SMA) elements, as considered in [18]. The authors considered applying the extended time-delayed feedback control on a SMA-pendulum system by exploring the SMA temperature-dependent behavior. The numerical results indicated that the controller was able to perform the system stabilization and that, after stabilization, control force tends to vanish.

In [19], Janzen et al. used the SMA in vibration control of a flexible link with slewing motion. The sliding mode control is considered for positioning control and vibration control of the flexible link. According to the authors, the use of the sliding mode control (SMC) is justified because the control technique has been commonly applied to nonlinear systems because of its robustness for uncertainties and external disturbances. Numerical simulations results are presented to demonstrate the effectiveness of the sliding mode strategy for the positioning control of the DC motor and for the vibration suppression of the flexible link by using SMA actuators.

In [20], the chaotic behavior of a micromechanical resonator is analyzed, and the control of chaos is projected. The analyses of the chaotic behavior considered phase portraits, maximum Lyapunov exponent, and bifurcation diagrams. Moreover, a robust fuzzy sliding mode controller (FSMC) was designed to turn the chaotic motion into a periodic motion even when the MEMS have parametrical uncertainties. Numerical simulations showed the robustness of the proposed control.

In the work, the 0-1 test and bifurcation diagrams are applied to investigate the nonlinear dynamics of the MEMS, considering the influence of the damping, variation of the polarization voltage, influence of the nonlinear stiffness term, and pull-in effect. Bifurcation diagrams were considered in this work for the identification of regular and chaotic behavior, as it is known to be useful and often used for systems with chaotic behavior, such as $[20,21]$. The $0-1$ test is a tool that makes it possible to analyze the results of the bifurcation diagram in a quantitative way and is successfully used in systems with chaotic behavior, such as [9-14]. In addition, in order to suppress the chaotic motion, the time-delayed feedback control technique and sliding model control are introduced.

This paper is organized as follows. Section 2 presents the mathematical model for microelectromechanical systems. In Section 3, nonlinearities are observed in the system through the $0-1$ test, considering the effects caused by the parameters, which are damping, polarization voltage, the nonlinear stiffness term, and $V_{i}$ for pull-in case. In Section 4 , the time-delayed feedback control is proposed and applied. In Section 5, the sliding mode control is proposed and applied. Finally, the paper is concluded in Section 6.

\section{Microelectromechanical System (MEMS)}

The behavior of the microelectromechanical system represented by an electrostatic generator of energy, shown in Figure 1, was studied previously in [22]. The physical system represented by Figure 1(a) may be considered as a set of microbeams [5]. The dynamical system under consideration is shown in Figure 1, and this device has two fixed plates and a movable plate between them (see Figure 1(b)), in which a voltage $V(t)$ composed of a polarization voltage (DC) $V_{p}$ and an alternating voltage (AC) $V_{i} \sin (w t)$ are applied. The DC voltage applies an electrostatic force on the beam and usually changes the equilibrium position. The plates have the function of providing electrodes to form a capacitor or storing electrical energy and provide elasticity or mechanical strength.

The equation of motion of the plates is given by

$$
m \ddot{x}=-F_{k}-F_{c}-F_{e},
$$

where $F_{k}$ is the conservative force of the spring, $F_{c}$ is the damping force of the elastic term, and $F_{e}$ is the electric force.

Note that, in Figure 1(b), the distance $d$ between the fixed and movable plates depends on the position of $x$ and $d_{0}$ (initial distance between the plates). Whereas the fixed plates have the same characteristics, the amount of total electric energy stored in the system can be obtained from

$$
\begin{aligned}
W^{*} & =\frac{\varepsilon_{0}}{2} V^{2} A\left(\frac{1}{d_{0}-x}\right)+\frac{\varepsilon_{0}}{2} V^{2} A\left(\frac{1}{d_{0}-x}\right) \\
& =\varepsilon_{0} A V^{2}\left(\frac{d_{0}}{d_{0}^{2}-x^{2}}\right) .
\end{aligned}
$$

Thus, the electric force $F_{e}$ is a nonlinear function of displacement in $x$ and a quadratic function of voltage:

$$
F_{e}=\frac{\partial W^{*}}{\partial x}=2 \varepsilon_{0} d_{0} A V^{2} \frac{x}{\left(d_{0}^{2}-x^{2}\right)^{2}} .
$$

The spring stiffness is also a parameter that can be affected by elastic term phenomena and nonlinearities, considering 


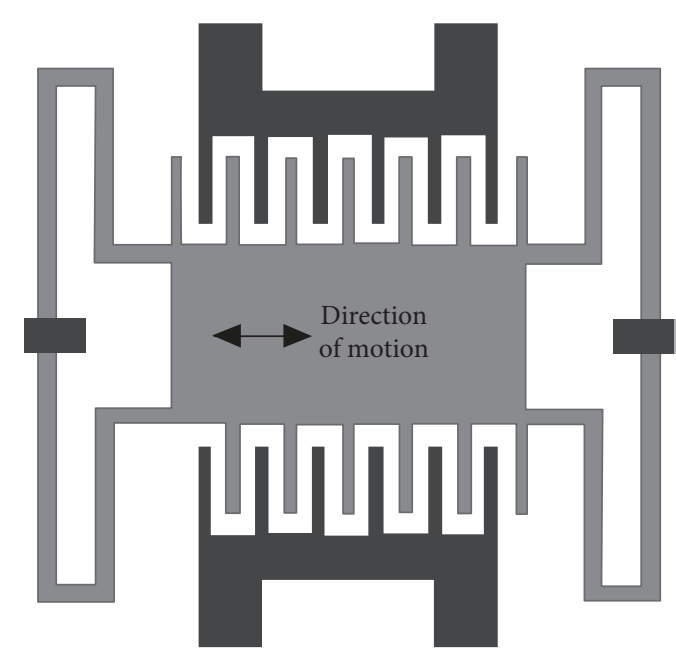

(a)

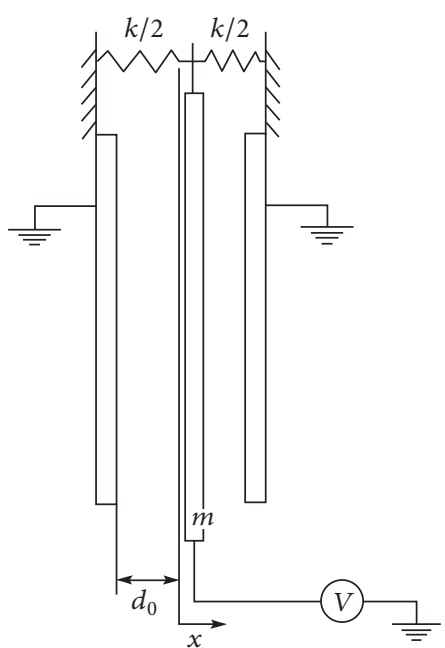

(b)

Figure 1: (a) In-plane gap closing. (b) Microelectromechanical system [5].

these variations. Then force $F_{k}$ of the conservative spring can be represented by

$$
F_{k}=k_{1} x+k_{3} x^{3}
$$

The dissipation force $F_{c}$ can be obtained from

$$
F_{c}=c \dot{x} .
$$

Substituting (3), (4), and (5) into (1), the equation of motion of the MEMS is obtained, given by

$$
m \ddot{x}+k_{1} x+k_{3} x^{3}+c \dot{x}=2 \varepsilon_{0} d_{0} A V^{2} \frac{x}{\left(d_{0}^{2}-x^{2}\right)^{2}} .
$$

Considering the initial conditions as $x(0)=x_{0}, \dot{x}(0)=\dot{x}_{0}$ and defining the new variables, $T=w_{0} t, u=x / x_{0},(6)$ can be represented in dimensionless form by

$$
\ddot{u}+\alpha_{1} u+\alpha_{3} u^{3}+b \dot{u}=\beta V^{2} \frac{u}{\left(d^{2}-u^{2}\right)^{2}},
$$

where $b=\left(c / m \omega_{0}\right), \alpha_{1}=\left(k_{1} / m \omega_{0}^{2}\right), \alpha_{3}=\left(k_{3} x_{0}^{2} / m \omega_{0}^{2}\right), \beta=$ $\left(2 \varepsilon_{0} d_{0} A / m \omega_{0}^{2} x_{0}^{4}\right), w=\omega / \omega_{0}$, and $d=d_{0} / x_{0}$.

Rewriting (7) in state-space notation, it has

$$
\begin{aligned}
& \dot{x}_{1}=x_{2} \\
& \dot{x}_{2}=-\alpha_{1} x_{1}-\alpha_{3} x_{1}^{3}-b x_{2}+\beta V^{2} \frac{x_{1}}{\left(d^{2}-x_{1}^{2}\right)^{2}},
\end{aligned}
$$

where $x_{1}=u$ and $x_{2}=\dot{u}$.

The equations of motion of the system are defined, and the next step is the dynamical analysis of the system, which is carried out in the next section.

\section{Dynamical Analysis Using 0-1 Test}

The $0-1$ test, proposed in $[9,10]$, is directly applied to a time series data, based on the statistical properties of a single coordinate; herein, the variable is $x_{1}$ (see (8)). Basically, the $0-1$ test consists of estimating a single parameter $K$. The test considers a system variable $x_{j}$, where two new coordinates $(p, q)$ are defined as follows:

$$
\begin{aligned}
& p(n, \bar{c})=\sum_{j=0}^{n} x(j) \cos (j \bar{c}) \\
& q(n, \bar{c})=\sum_{j=0}^{n} x(j) \sin (j \bar{c}),
\end{aligned}
$$

where $\bar{c} \in(0, \pi)$ is a constant. The mean square displacement of the new variables $p(n, \bar{c})$ and $q(n, \bar{c})$ is given by

$$
\begin{aligned}
& M(n, c)=\lim _{n \rightarrow \infty} \frac{1}{N} \sum_{j=1}^{N}\left[(p(j+n, \bar{c})-p(j, \bar{c}))^{2}\right. \\
& \left.+(q(j+n, \bar{c})-q(j, \bar{c}))^{2}\right],
\end{aligned}
$$

where $n=1,2, \ldots, N$ and, therefore, the parameter $K_{c}$ is obtained in the limit of a very long time by

$$
K=\frac{\operatorname{cov}(Y, M(\bar{c}))}{\sqrt{\operatorname{var}(Y) \operatorname{var}(M(\bar{c}))}},
$$

where vectors $M(\bar{c})=\left[M(1, \bar{c}), M(2, \bar{c}), \ldots, M\left(n_{\max } \bar{c}\right)\right]$ and $Y=1,2, \ldots, n_{\max }$.

Given any two vectors $x$ and $y$, the covariance $\operatorname{cov}(x, y)$ and variance $\operatorname{var}(x)$, of $n_{\max }$ elements, are usually defined as [11]

$$
\begin{aligned}
\operatorname{cov}(x, y) & =\frac{1}{n_{\max }} \sum_{n=1}^{n_{\max }}(x(n)-\bar{x})(y(n)-\bar{y}) \\
\operatorname{var}(x) & =\operatorname{cov}(x, y),
\end{aligned}
$$

where $\bar{x}$ and $\bar{y}$ are the average of $x(n)$ and $y(n)$, respectively. As a final result, the value of the searched parameter $K$ is 


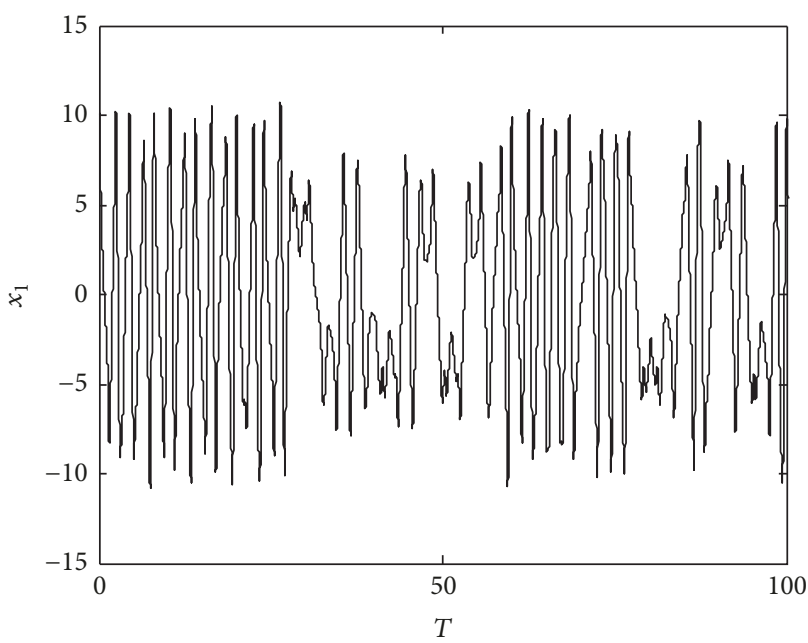

(a)

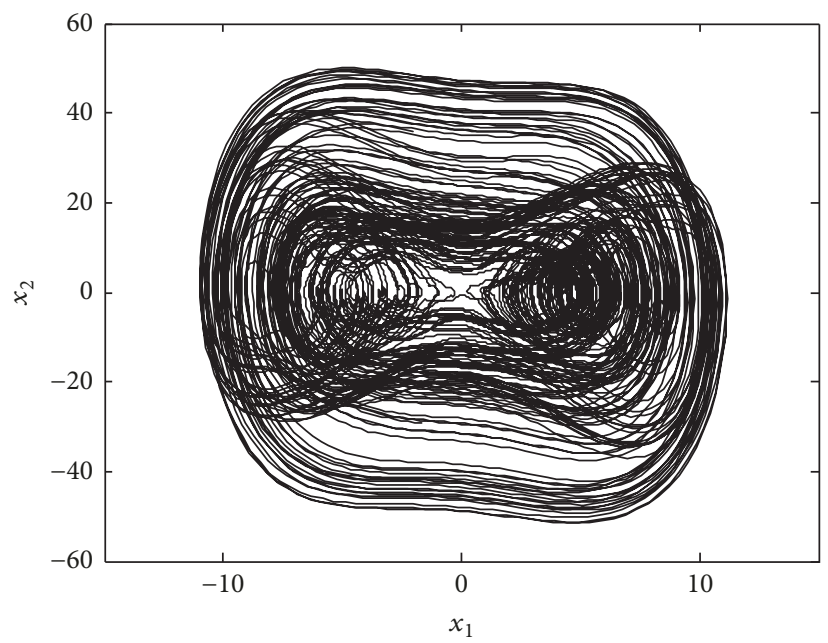

(b)

Figure 2: (a) Displacement. (b) Phase plane.

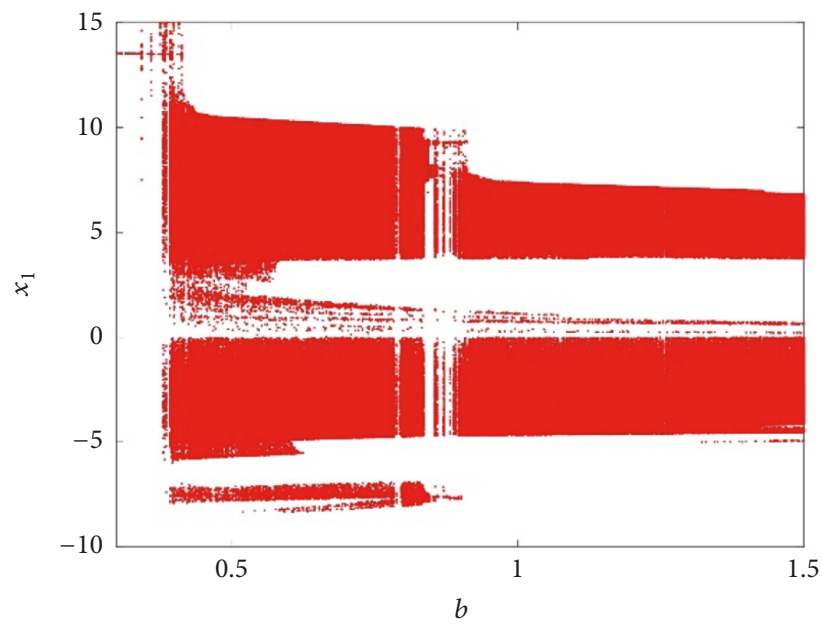

(a)

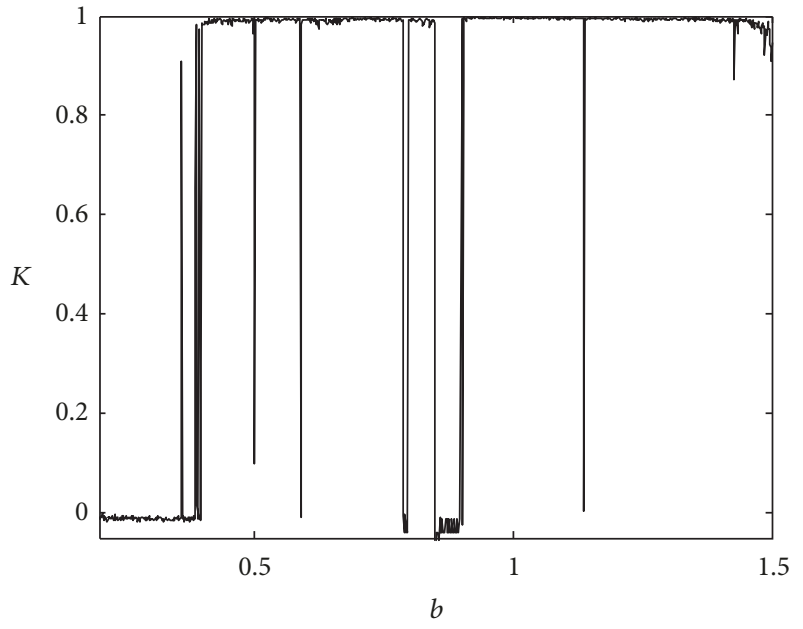

(b)

Figure 3: (a) Bifurcation diagram ( $b$ versus $x_{1}$ ). (b) $K$ versus $b$.

obtained taking the median of 100 different values of the parameter $\bar{c} \in(0, \pi)$ in (11). If $K$ value is close to 0 , the system is periodic; on the other hand, if $K$ value is close to 1 , the system is chaotic. In all simulations, $n=1000$ and $j=n / 100, \ldots, n / 10$ were chosen.

The values of the parameters used in the current numerical investigations were taken from [23] and they are as follows: $\alpha_{1}=1, \alpha_{3}=0.4, \beta=69141.6, b=0.5, d=25$, $\omega=6.28, V_{p}=2, V_{i}=10, x_{10}=1$, and $x_{20}=1.5$. The parameters $\alpha_{1}, \alpha_{3}, \beta$, and $b$ were chosen in [16] such as $\left(k_{1} \ll\right.$ $\left.2 \varepsilon_{0} d_{0} A\right)$.

The time history of the displacement and the phase plane can be observed in Figure 2.

Next, the effects caused by the parameters will be considered: damping parameter $b$, the polarization voltage $V_{p}$, the nonlinear stiffness term $\alpha_{3}$, and $V_{i}$ for pull-in case.
3.1. Damping Effect of the MEMS. The numerical study of the MEMS shows that the damping effect can be very significant. Figure 3 shows the bifurcation diagram and the $0-1$ test varying the damping parameter $b$, considering $b \in[0.2,1.5]$. As can be seen in Figure 3, the system is periodic to the left of $b \approx 0.4$ and most of resting values of $b$ the system are chaotic.

A tendency of stability of chaotic behavior with the damping $b$ variation is presented in Figure 3, indicating that a significant region of the parameter $b$ led the system to chaotic behavior. Periodic behaviors are observed in small regions of parameter $b$, which are the intervals $0.2 \leq b<0.35$ or $0.849<b<0.896$.

3.2. Influence of the Polarization Voltage. Consider now the variation of the polarization voltage $V_{p}$ of the nonlinear MEMS. Figure 4 shows the bifurcation diagram and the 0-1 


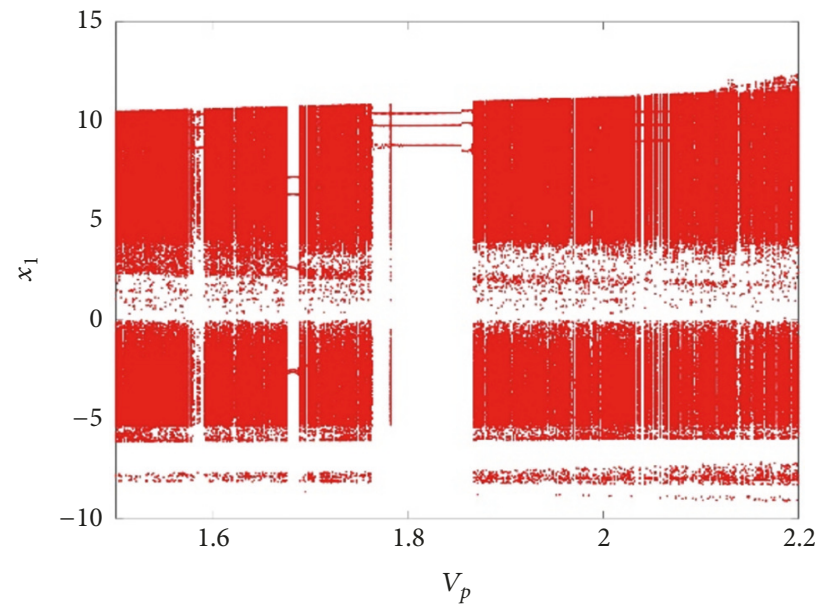

(a)

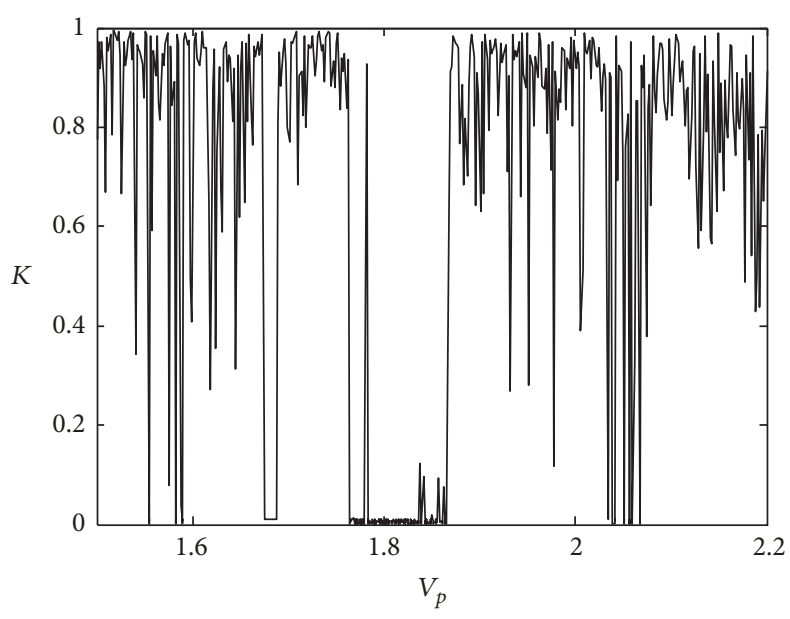

(b)

Figure 4: (a) Bifurcation diagram $\left(V_{p}\right.$ versus $\left.x_{1}\right)$. (b) $K$ versus $V_{p}$.

test. As can be seen in Figure 4 for the considered range $V_{p} \epsilon$ $[1.5,2.5]$, a large variety of periodic and chaotic responses are observed.

Different from the observed behavior for the parameter $b$, it is possible to see in Figure 4 a tendency to instability of chaotic behavior to variations of $V_{p}$, indicating many areas of short intervals where the system maintains the chaotic behavior. Then periodic behaviors are encountered in the intervals of parameter $V_{p}$ that are $1.676<V_{p}<1.686,1.765<$ $V_{p}<1.78$, or $1.784<b<1.865$.

3.3. Influence of the Nonlinear Stiffness Term. Now, the variation of the nonlinear stiffness term $\alpha_{3}$ is considered, considering $a_{3} \in[0.35,1.5]$. The same bifurcation diagram and $0-1$ test analyses were carried out and presented in Figure 5.

Analyzing the bifurcation diagram (Figure 5(a)) and 0-1 test (Figure 5(b)), it is possible to observe that there are two regions where the variation of $\alpha_{3}$ leads to periodic behavior. Such intervals are $0.79<\alpha_{3}<1.12$ and $1.34<\alpha_{3}<1.5$. In the other areas, the system presented stability of chaotic behavior. Considering those results, it can be seen that the parameter $\left(\alpha_{3}\right)$ is the parameter with larger interval when $K=$ 0 , demonstrating that the appearance of chaotic behavior in this system is more sensitive to variation of this parameter.

3.4. Pull-In. The electric charge acting on a capacitor plate is composed of a continuous bias voltage and an alternating voltage. The DC component applies an electric force to a plate, thereby diverting it to a new equilibrium position, while an $\mathrm{AC}$ component vibrates the plate around the equilibrium position. The combined electric charge thus leads to a continuous increase in the deflection of the microstructure and, accordingly, an increase in the electrical forces in a positive feedback loop. This behavior continues until a physical contact is made with the stationary electrode. This phenomenon of structural instability is known as pull-in.

Now, the variation of the alternating voltage $\left(V_{i}\right)$ is considered, considering $V_{i} \in[0,10]$.
Figure 6 shows a bifurcation diagram varying $V_{i}$.

Figure 6 shows the region in which the pull-in effect occurs, which is when $V_{i} \approx 10$.

\section{Time-Delayed Feedback Control}

As originally suggested by the author of [24], continuous control input $U$ stabilizing a chaotic oscillation is given by the difference between the current output and the past one as follows [17-19].

$$
\begin{aligned}
& U \\
& =\kappa\left\{g\left[x_{1}(T-\tau), x_{2}(T-\tau)\right]-g\left[x_{1}(T), x_{2}(T)\right]\right\},
\end{aligned}
$$

where $\tau$ is the time delay and $k$ the feedback gain.

The terms $g\left[x_{1}(T-\tau), x_{2}(T-\tau)\right]$ and $g\left[x_{1}(T), x_{2}(T)\right]$ represent scalar output signals measured at the current time $T$ and at the previous time $(T-\tau)$, respectively $[16,17,24-$ 26]. Since the control input (13) only depends on the output signal, the time delay $\tau$ is adjusted to the period of a target unstable periodic orbit that is intended to be stabilized in a chaotic attractor, and the control input therefore converges to null after the controlled system is stabilized to the target orbit.

Assuming that the velocity of oscillation is measured as an output of nonlinear system (7), the control signal $U$ is given as follows:

$$
U=\kappa\left[x_{2}(T-\tau)-x_{2}(T)\right]
$$

The MEMS with control signal (14) is expressed in the following way:

$$
\begin{aligned}
\dot{x}_{1}= & x_{2} \\
\dot{x}_{2}= & -\alpha_{1} x_{1}-\alpha_{3} x_{1}^{3}-b x_{2}+\beta V^{2} \frac{x_{1}}{\left(d^{2}-x_{1}^{2}\right)^{2}} \\
& +\kappa\left[x_{2}(T-\tau)-x_{2}(T)\right] .
\end{aligned}
$$




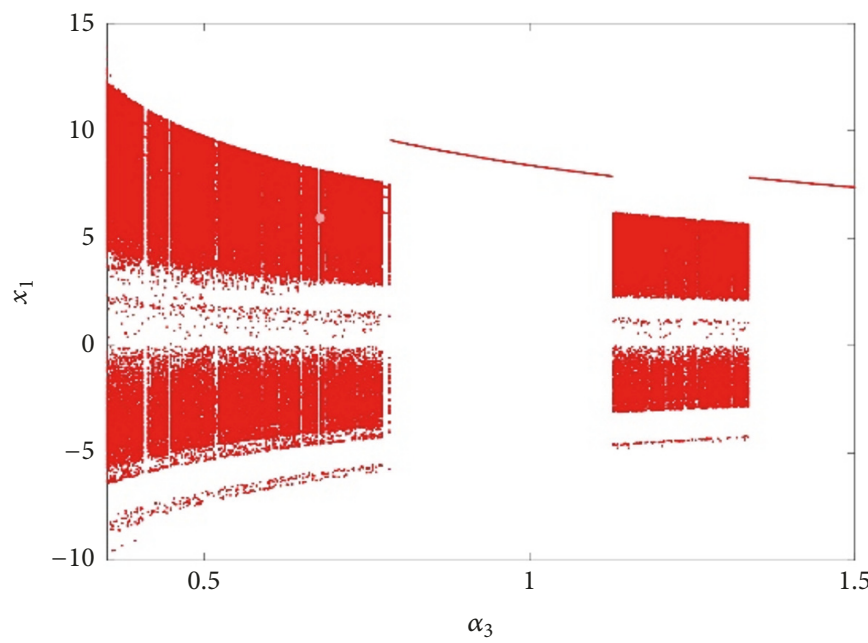

(a)

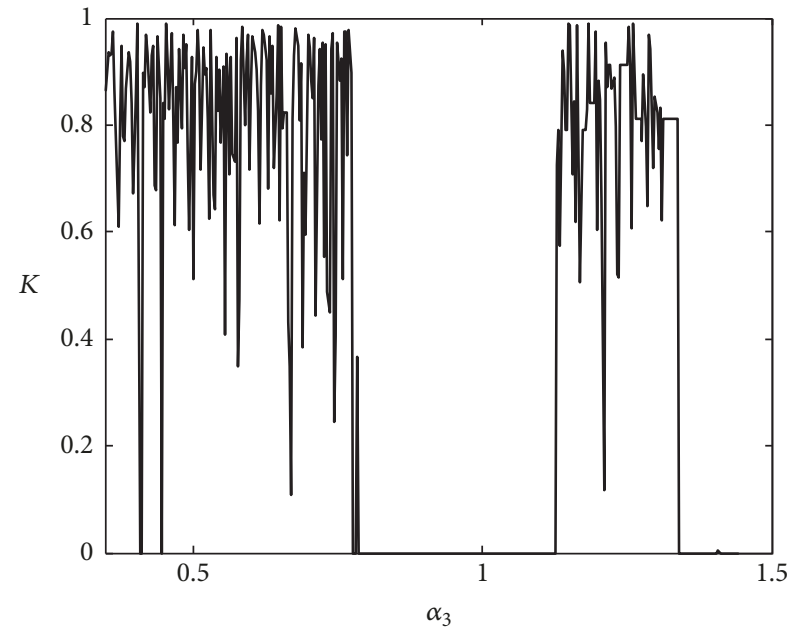

(b)

FIgURE 5: (a) Bifurcation diagram $\left(\alpha_{3}\right.$ versus $\left.x_{1}\right)$. (b) $\alpha_{3}$ versus $K$.

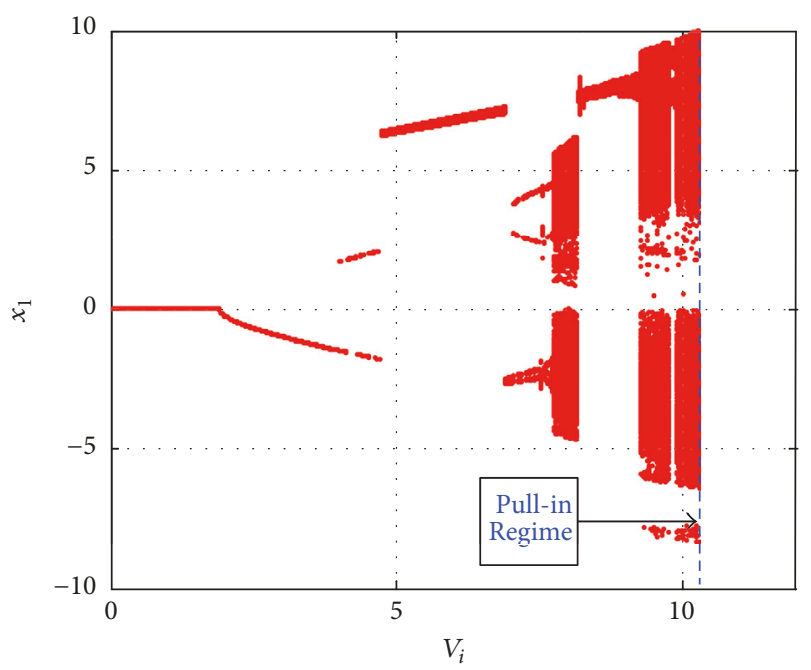

FIGURE 6: Bifurcation diagram with pull-in.

The time delay $\tau$ and feedback gain $\kappa$ are important control parameters that substantially affect the control performance. The time delay $\tau$ is adjusted to $\tau=2 \pi / \omega \approx 1$ to stabilize an orbit with the same frequency as the external force oscillating the system. Then, firstly, a variation of the control gain was carried out in Figure 7 in order to observe the behavior of the system. The system is chaotic from 0 to 0.3 approximately. After that, the behavior of the system becomes periodic.

Choose $\kappa=0.35$, that is, a periodic behavior, Figure 8 shows the application of control (14).

It can be observed in Figure 8(c) that the time-delayed control was efficient in leading the system to a periodic orbit. However, for the cases where it is desired to take the system to a previously defined orbit, the control may not be the most suitable. For these mentioned cases, in the next section the sliding mode control will be considered.

\section{Proposed Sliding Mode Control (SMC)}

As can be seen in the previous section, the time delay control took the system from a chaotic behavior to a periodic attractor of the system. In this section, the case of imposing the desired orbit where the time delay control cannot be applied will be considered. For this case the sliding mode control is considered.

Consider now (8) in the form

$$
\begin{aligned}
& \dot{x}_{1}=x_{2} \\
& \dot{x}_{2}=-\alpha_{1} x_{1}-\alpha_{3} x_{1}^{3}-b x_{2}+\beta V^{2} \frac{x_{1}}{\left(d^{2}-x_{1}^{2}\right)^{2}}+U,
\end{aligned}
$$

where $U$ is sliding mode control signal.

Define the desired trajectory errors as

$$
e=\left[\begin{array}{l}
x_{1}-x_{1}^{*} \\
x_{2}-x_{2}^{*}
\end{array}\right]
$$

where $x_{1}^{*}$ is a desired trajectory; the dynamic equations of these errors (17) can be obtained as

$$
\begin{aligned}
\dot{e}_{1}= & e_{2} \\
\dot{e}_{2}= & -\alpha_{1}\left(e_{1}-x_{1}^{*}\right)-\alpha_{3}\left(e_{1}-x_{1}^{*}\right)^{3}-b\left(e_{1}-x_{2}^{*}\right) \\
& +\beta V^{2} \frac{\left(e_{1}-x_{1}^{*}\right)}{\left(d^{2}-\left(e_{1}-x_{1}^{*}\right)\right)^{2}}+U .
\end{aligned}
$$

For the sliding mode control field, the sliding surface is generally taken to be $[19,20,27]$

$$
s=e_{1}-\lambda e_{2} .
$$

The existence of the sliding mode requires the following conditions to be satisfied:

$$
\begin{aligned}
& s=e_{1}-\lambda e_{2} \\
& \dot{s}=\dot{e}_{1}-\lambda \dot{e}_{2},
\end{aligned}
$$

where $\lambda$ represents a real number. 


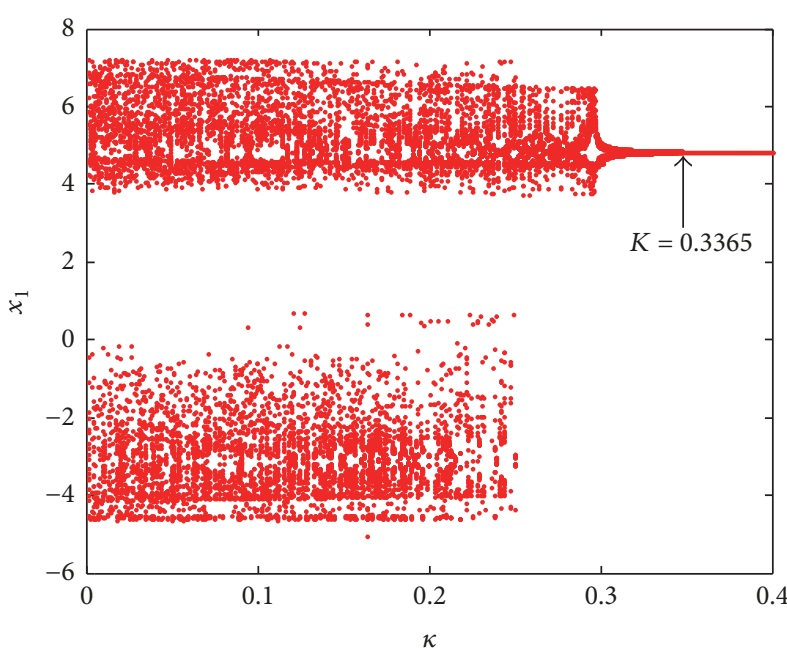

(a)

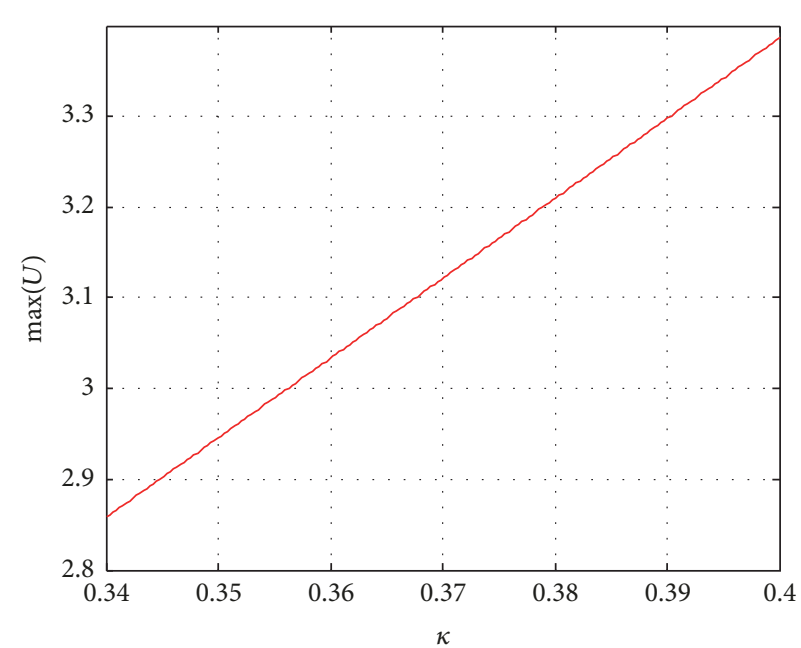

(b)

FIGURE 7: (a) Bifurcation diagram for control gain $(\kappa)$. (b) Variation of the control signal $U$ for the gain variation $(\kappa)$.

Equation (19) defines the output of the sliding mode control controller, while the reaching law is given by [27]

$$
U= \begin{cases}U_{\max } & \text { if } \frac{s}{\phi}<-1 \\ -U_{\max } & \text { if } \frac{s}{\phi}>1 \\ K_{c} s & \text { if }-1<\frac{s}{\phi}<1,\end{cases}
$$

where $\phi$ is layer thickness of the control, $K$ is a proportional gain, and $U_{\max }$ is control of the saturation value.

For $K_{c}$ determination, the Lyapunov function of the system is considered, defined as

$$
V=\frac{1}{2} s^{2}
$$

The first derivative of this system with respect to time can be expressed as

$$
\begin{aligned}
\dot{V} & =\dot{s} s=s\left[\dot{e}_{2}+\lambda \dot{e}_{1}\right]=s\left[-\alpha_{1}\left(e_{1}-x_{1}^{*}\right)\right. \\
& -\alpha_{3}\left(e_{1}-x_{1}^{*}\right)^{3}-b\left(e_{1}-x_{2}^{*}\right) \\
& \left.+\beta V^{2} \frac{\left(e_{1}-x_{1}^{*}\right)}{\left(d^{2}-\left(e_{1}-x_{1}^{*}\right)\right)^{2}}+K U+\lambda e_{2}\right] \cdot=s\left[K_{c} U\right] \\
& \leq-K_{c}|s|
\end{aligned}
$$

If $K_{c}>0$ is selected, then the reaching condition $(s \dot{s}<0)$ is always satisfied. Therefore, system (8) can be stabilized to a desired trajectory $x_{1}^{*}(T)$.
Defining the desired orbits to periodic orbits obtained with the time delay control of Figure 8(c), they are obtained through the use of Fourier series calculated numerically as

$$
\begin{aligned}
x_{1}^{*}= & 4.099049-0.80068 \cos (2 \pi T) \\
& +0.123057 \sin (2 \pi T)-0.22686 \cos (4 \pi T) \\
& -0.06764 * \sin (4 \pi T)+0.016746 \cos (6 \pi T) \\
& +0.002594 \sin (6 \pi T)+0.001333 \cos (8 \pi T) \\
& +0.001167 \sin (8 \pi T) \\
x_{2}^{*}= & 0.060495+0.773184 \cos (2 \pi T) \\
& +5.030938 \sin (2 \pi T)-0.85001 \cos (4 \pi T) \\
& +2.850889 \sin (4 \pi T)+0.048871 \cos (6 \pi T) \\
& -0.31566 \sin (6 \pi T)+0.029304 \cos (8 \pi T) \\
& -0.0335 \sin (8 \pi T)
\end{aligned}
$$

and the parameters $\lambda=4, K_{c}=1000, \phi=10^{-3}$, and $U_{\max }=500$ are considered. Figure 9 shows the application of the sliding mode control in the MEMS problem.

As can be seen in Figure 9(b), the proposed control (21) was efficient and it led system (16) from the initial state $x_{1_{0}}$ to desired state $x_{1}^{*}$.

Figure 9(a) shows the difference between the state obtained with the control $U$ and the desired state $x_{1}^{*}$, as it is seen that the control took approximately $1.65 \mathrm{~T}$ to stabilize the system in the desired orbit. In addition, Figure 9(c) presented the phase portrait for controlled systems (16), demonstrating that the system is periodic.

\section{Conclusions}

In this work, the dynamical analysis and control of the MEMS problem were investigated and discussed, contributing to 


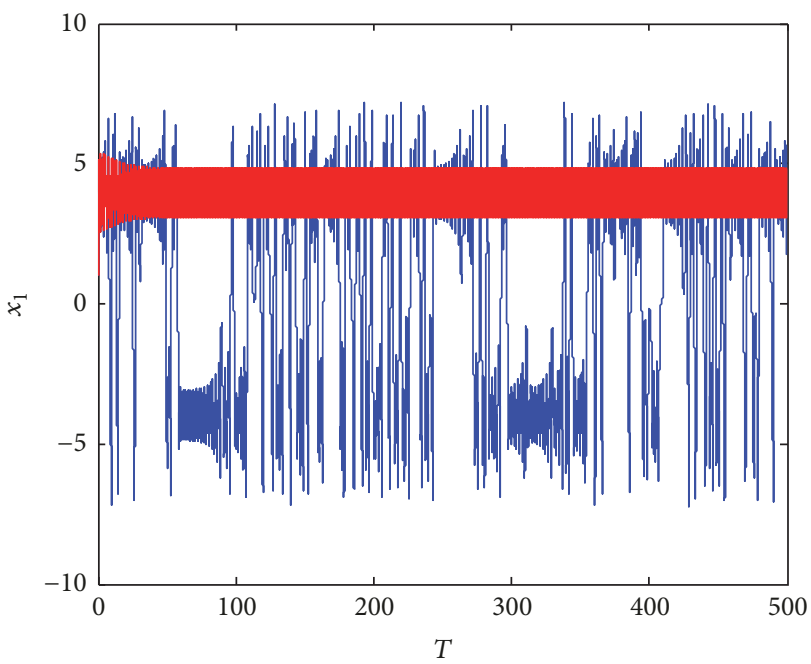

_ without control with control

(a)

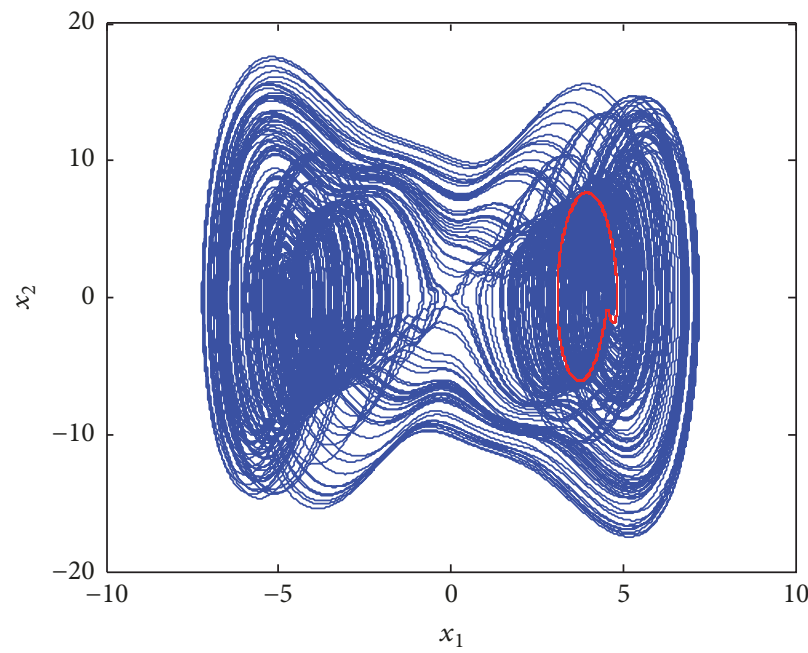

- without control

(c)

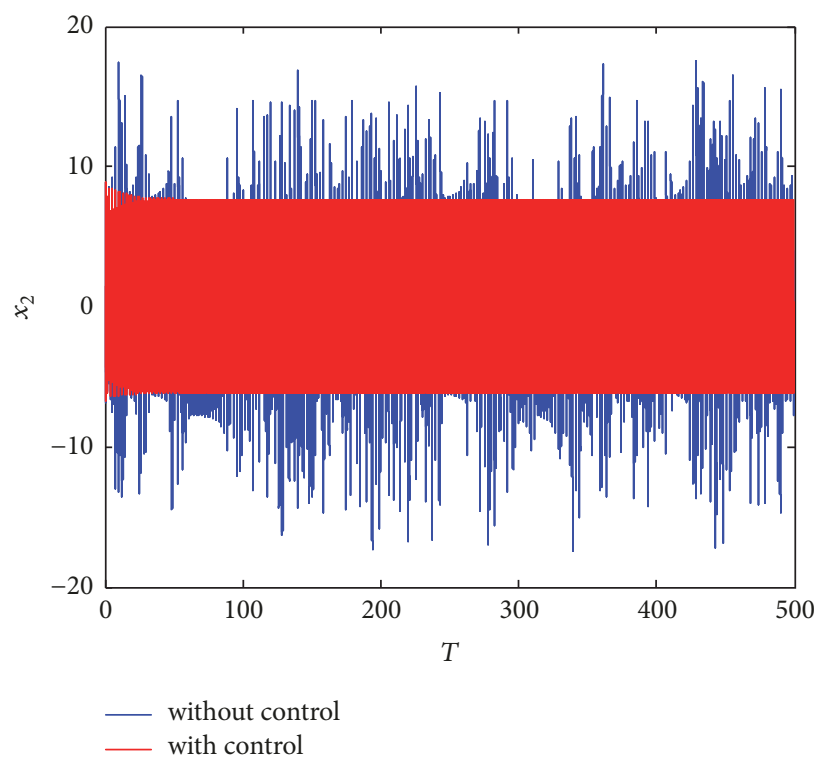

(b)

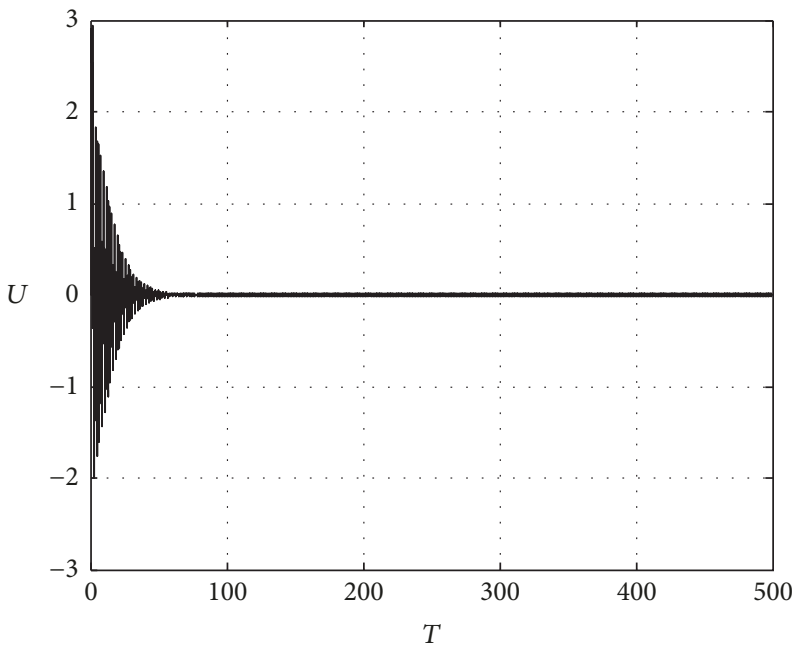

(d)

Figure 8: (a) Displacement. (b) Velocity. (c) Phase portrait. (d) Control signal.

the analysis of the influence of the parameters (damping coefficient, polarization of the voltage, and nonlinear stiffness term) and the design of two control techniques to control the chaotic behavior of the MEMS. With the presented numerical results, it is expected that such results contribute to new information that supports the physical assembly of a MEMS with behaviors close to those desired or for the cases of control application that can contribute to the control project.

Numerical simulations showed the existence of chaotic behavior for some regions in the parameter space of the damping, the polarization voltage, and the nonlinear stiffness term. Chaos was characterized by applying $0-1$ test. The bifurcation diagrams were constructed to explore the qualitative behavior of the system.
In order to suppress the chaotic motion, the time-delayed feedback control and sliding mode control techniques were projected. The efficiency of the technique was demonstrated through numerical simulations in order to eliminate the chaotic behavior of the system. As could be seen in Figure $8(\mathrm{c})$, the time-delayed control led the system to one of the periodic attractors of the system, and we only used the control signal until the system stabilizes in the periodic orbit (see Figure 8(d)).

As the time-delayed control is not designed to take the system to any previously defined orbit, an alternative is the sliding mode control application. As could be seen in Figure 9(c), the sliding mode control was efficient in taking the system to the same periodic orbit obtained by the 


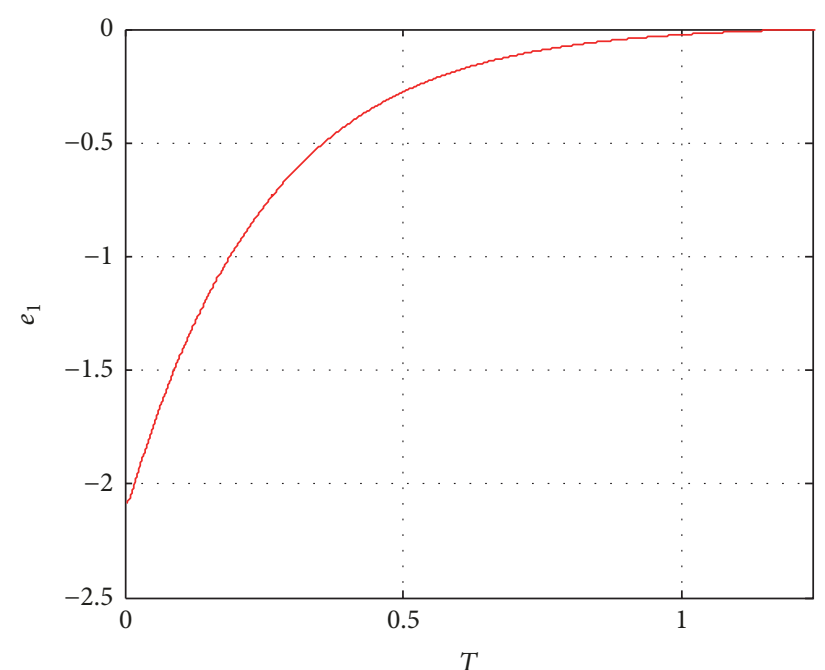

(a)

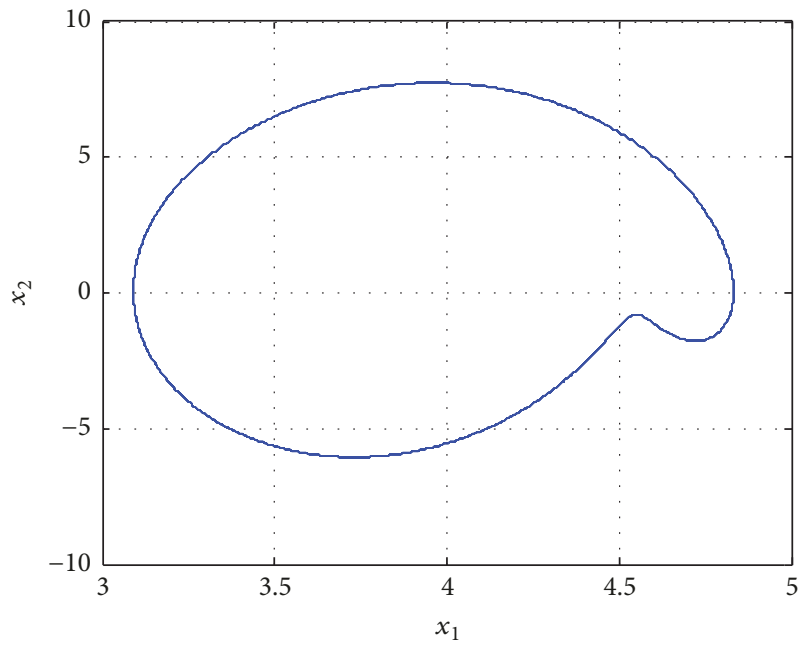

(c)

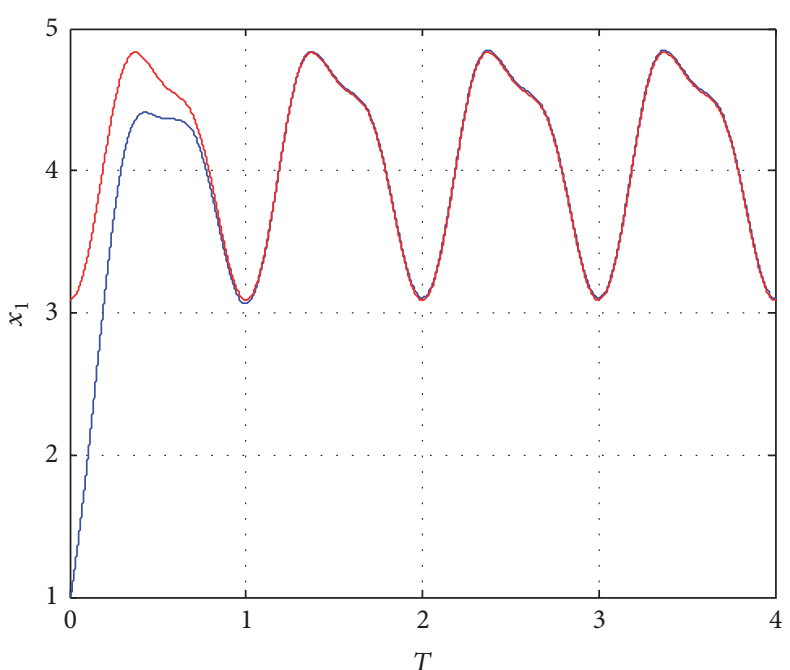

$x_{1}$
$-x_{1}^{*}$

(b)

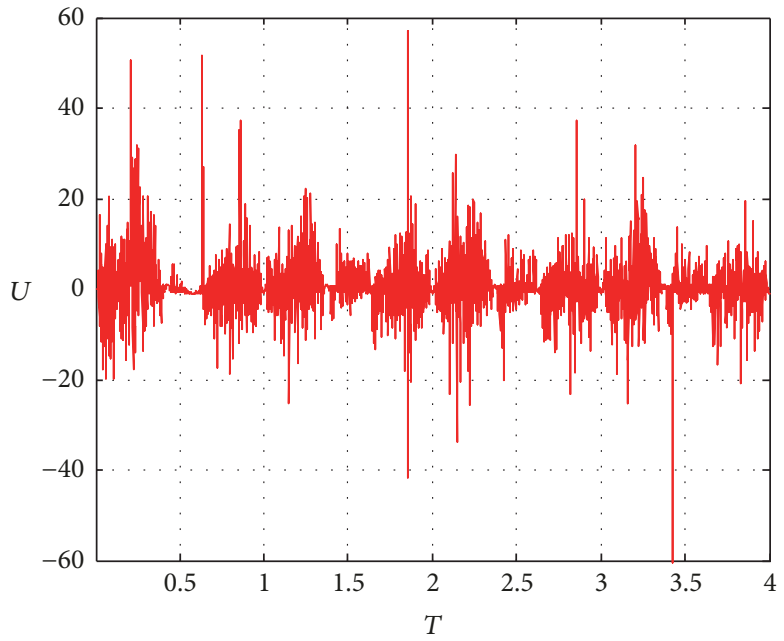

(d)

FiguRe 9: (a) Desired trajectory error $\left(e_{1}\right)$. (b) Displacement $\left(x_{1}^{*}\right.$ is the desired trajectory and $x_{1}$ is displacement with proposed control (20)). (c) Phase portrait for controlled systems. (d) Control signal.

time-delayed control. However, when the control signals of Figures $8(\mathrm{~d})$ and $9(\mathrm{~d})$ are compared with each other, it can be observed that the sliding mode control has to maintain a control signal to keep the system in the periodic orbit, generating a control cost superior to the control cost of the time-delayed control.

Thus, it is possible to conclude that the time delay is an excellent option when the objective is to take the system to a periodic orbit with the lowest cost of control. Furthermore, the sliding mode control is an option to be considered in order to impose the desired orbit.

In future works, the efficiency of control techniques will be compared to dynamical analyses of the system with fractional-order derivatives. In addition, the analysis of the applicability of the control in an analogous experimental model is carried out, considering the control by the electrical voltage similar to that used in [15] or the control through the use of intelligent materials (SMA) as used in $[18,19]$.

\section{Data Availability}

The data used to support the findings of this study are available from the corresponding author upon request.

\section{Conflicts of Interest}

The authors declare that there are no conflicts of interest regarding the publication of this paper. 


\section{Acknowledgments}

The authors acknowledge support by $\mathrm{CNPq}$ (Grant 447539 / 2014-0), CAPES, and all Brazilian research funding agencies.

\section{References}

[1] D. B. Blocher, A. T. Zehnder, and R. H. Rand, "Entrainment of micromechanical limit cycle oscillators in the presence of frequency instability," Journal of Microelectromechanical Systems, vol. 22, no. 4, Article ID 6478863, pp. 835-845, 2013.

[2] D. Blocher, R. H. Rand, and A. T. Zehnder, "Analysis of laser power threshold for self oscillation in thermo-optically excited doubly supported MEMS beams," International Journal of NonLinear Mechanics, vol. 57, pp. 10-15, 2013.

[3] M. Pandey, R. H. Rand, and A. T. Zehnder, "Frequency locking in a forced Mathieu-van der Pol-Duffing system," Nonlinear Dynamics, vol. 54, no. 1-2, pp. 3-12, 2008.

[4] K. Aubin, M. Zalalutdinov, T. Alan et al., "Limit cycle oscillations in CW laser-driven NEMS," Journal of Microelectromechanical Systems, vol. 13, no. 6, pp. 1018-1026, 2004.

[5] A. M. Tusset, J. M. Balthazar, D. G. Bassinello, J. Pontes, and J. L. Felix, "Statements on chaos control designs, including a fractional order dynamical system, applied to a MEMS comb-drive actuator," Nonlinear Dynamics, vol. 69, no. 4, pp. 1837-1857, 2012.

[6] A. M. Tusset, Á. M. Bueno, C. B. Nascimento, M. Dos Santos Kaster, and J. M. Balthazar, "Nonlinear state estimation and control for chaos suppression in MEMS resonator," Shock and Vibration, vol. 20, no. 4, pp. 749-761, 2013.

[7] S. Sabarathinam and K. Thamilmaran, "Implementation of analog circuit and study of chaotic dynamics in a generalized Duffing-type MEMS resonator," Nonlinear Dynamics, vol. 87, no. 4, pp. 2345-2356, 2017.

[8] H. S. Haghighi and A. H. D. Markazi, "Chaos prediction and control in MEMS resonators," Communications in Nonlinear Science and Numerical Simulation, vol. 15, no. 10, pp. 3091-3099, 2010.

[9] G. A. Gottwald and I. Melbourne, "A new test for chaos in deterministic systems," Proceedings of the Royal Society A Mathematical, Physical and Engineering Sciences, vol. 460, no. 2042, pp. 603-611, 2004.

[10] G. A. Gottwald and I. Melbourne, "Testing for chaos in deterministic systems with noise," Physica D: Nonlinear Phenomena, vol. 212, no. 1-2, pp. 100-110, 2005.

[11] D. Bernardini and G. Litak, "An overview of 0-1 test for chaos," Journal of the Brazilian Society of Mechanical Sciences and Engineering, vol. 38, no. 5, pp. 1-8, 2015.

[12] D. Bernardini, G. Rega, G. Litak, and A. Syta, "Identification of regular and chaotic isothermal trajectories of a shape memory oscillator using the 0-1 test," Proceedings of the Institution of Mechanical Engineers, Part K: Journal of Multi-body Dynamics, vol. 227, no. 1, pp. 17-22, 2013.

[13] G. Litak, A. Syta, and M. Wiercigroch, "Identification of chaos in a cutting process by the 0-1 test," Chaos, Solitons \& Fractals, vol. 40, no. 5, pp. 2095-2101, 2009.

[14] G. Litak, D. Bernardini, A. Syta, G. Rega, and A. Rysak, "Analysis of chaotic non-isothermal solutions of thermomechanical shape memory oscillators," The European Physical Journal Special Topics, vol. 222, no. 7, pp. 1637-1647, 2013.

[15] K. Pyragas and A. Tamaševičius, "Experimental control of chaos by delayed self-controlling feedback," Physics Letters A, vol. 180, no. 1-2, pp. 99-102, 1993.
[16] J. M. Balthazar, A. M. Tusset, S. L. T. De Souza, and A. M. Bueno, "Microcantilever chaotic motion suppression in tapping mode atomic force microscope," Proceedings of the Institution of Mechanical Engineers, Part C: Journal of Mechanical Engineering Science, vol. 227, no. 8, pp. 1730-1741, 2013.

[17] K. D. S. Rodrigues, J. M. Balthazar, A. M. Tusset, B. R. de Pontes, and Á. M. Bueno, "Preventing Chaotic Motion in TappingMode Atomic Force Microscope," Journal of Control, Automation and Electrical Systems, vol. 25, no. 6, pp. 732-740, 2014.

[18] D. D. Costa and M. A. Savi, "Chaos control of an SMApendulum system using thermal actuation with extended timedelayed feedback approach," Nonlinear Dynamics, pp. 1-13, 2018.

[19] F. C. Janzen, J. M. Balthazar, A. M. Tusset, R. T. Rocha, and J. J. De Lima, "Angular positioning and vibration control of a slewing flexible control by applying smart materials and sliding modes control," in Proceedings of the ASME 2017 International Design Engineering Technical Conferences and Computers and Information in Engineering Conference, IDETC/CIE 2017, vol. 8, Cleveland, Oh, USA, August 2017.

[20] H.-T. Yau, C.-C. Wang, C.-T. Hsieh, and C.-C. Cho, "Nonlinear analysis and control of the uncertain micro-electro-mechanical system by using a fuzzy sliding mode control," Computers \& Mathematics with Applications. An International Journal, vol. 61, no. 8, pp. 1912-1916, 2011.

[21] C.-C. Wang, "Non-periodic and chaotic response of threemultilobe air bearing system," Applied Mathematical Modelling: Simulation and Computation for Engineering and Environmental Systems, vol. 47, pp. 859-871, 2017.

[22] F. C. Moon, Applied Dynamics-With Applications to Multibody and Mechatronic Systems, Wiley-Interscience, New York, NY, USA, 1998.

[23] D. G. Bassinello, J. M. Balthazar, A. M. Tusset, and V. Piccirillo, "Dynamic Analysis of a Fractional-Order MEMS System Using 0-1 Test," in Proceedings of the ASME 2016 International Design Engineering Technical Conferences and Computers and Information in Engineering Conference, pp. V008T10A014V008T10A014, 2016.

[24] K. Pyragas, "Continuous control of chaos by self-controlling feedback," Physics Letters A, vol. 170, no. 6, pp. 421-428, 1992.

[25] K. Yamasue and T. Hikihara, "Control of microcantilevers in dynamic force microscopy using time delayed feedback," Review of Scientific Instruments, vol. 77, no. 5, Article ID 053703, pp. 1-6, 2006.

[26] H. Salarieh and A. Alasty, "Control of chaos in atomic force microscopes using delayed feedback based on entropy minimization," Communications in Nonlinear Science and Numerical Simulation, vol. 14, no. 3, pp. 637-644, 2009.

[27] V. I. Utkin, Sliding Modes in Control Optimization, SpringerVerlag, Berlin, Germany, 1992. 


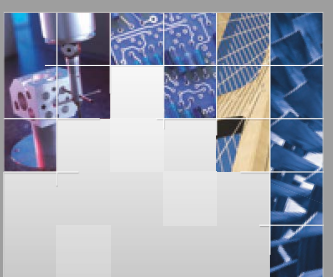

\section{Enfincering}
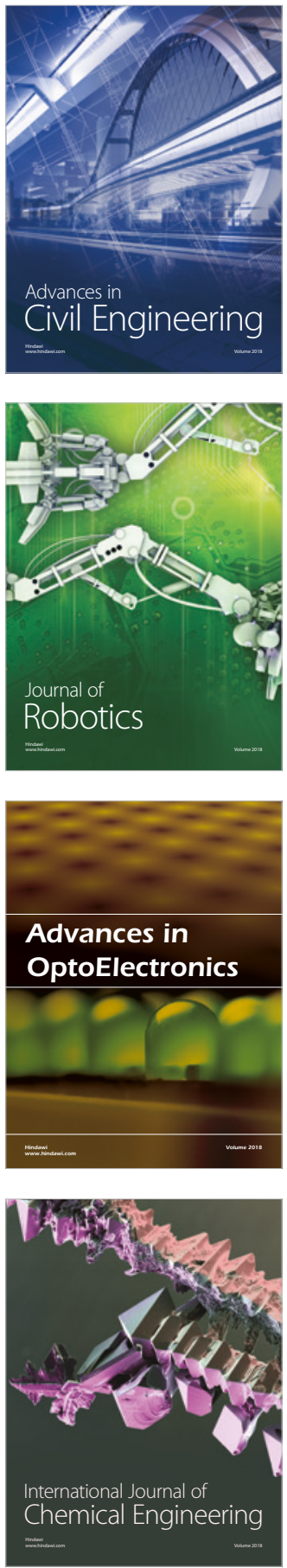

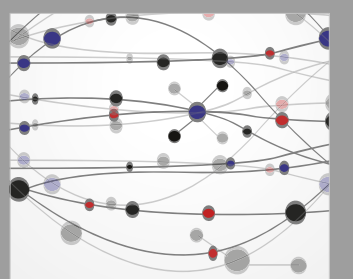

\section{Rotating \\ Machinery}

The Scientific World Journal

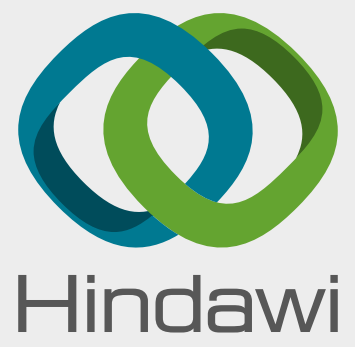

Submit your manuscripts at

www.hindawi.com
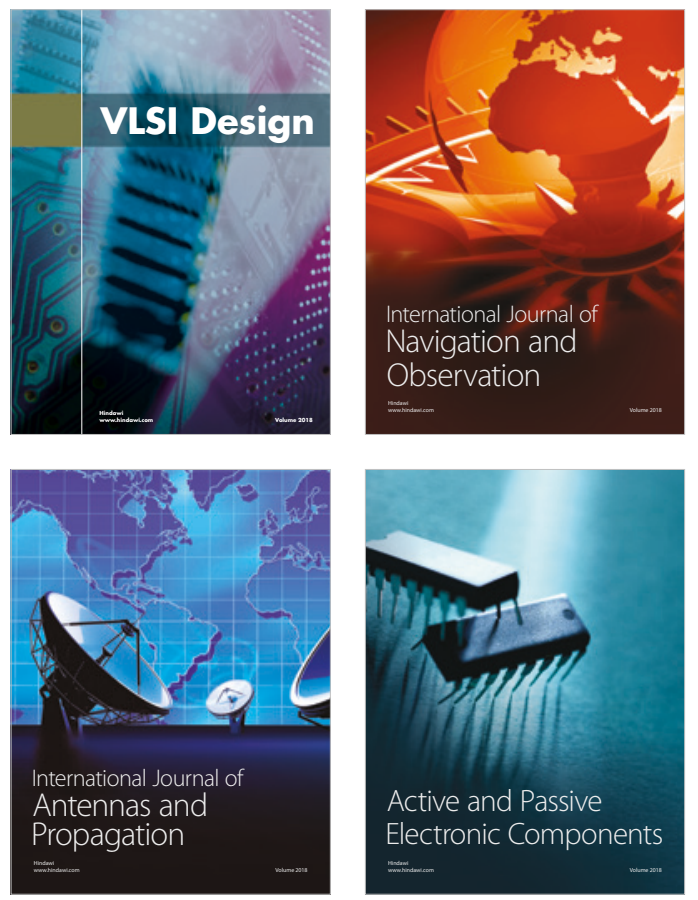
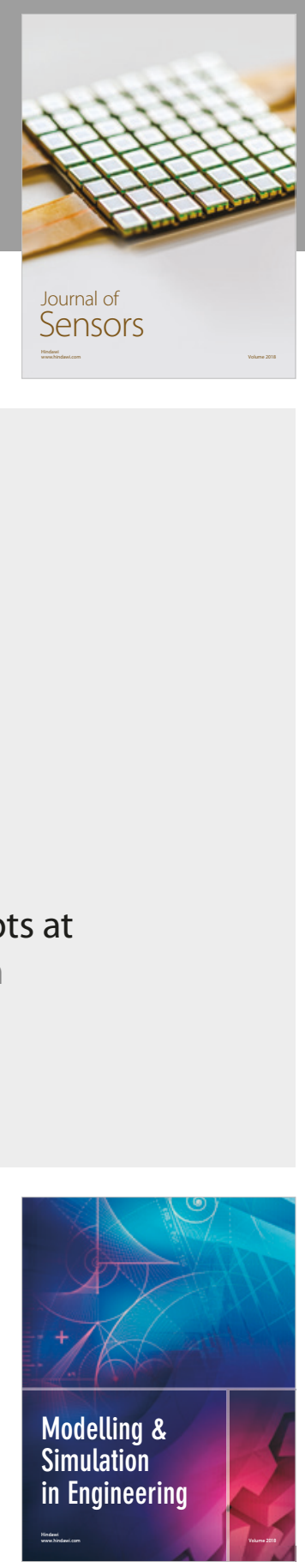

\section{Advances \\ Multimedia}
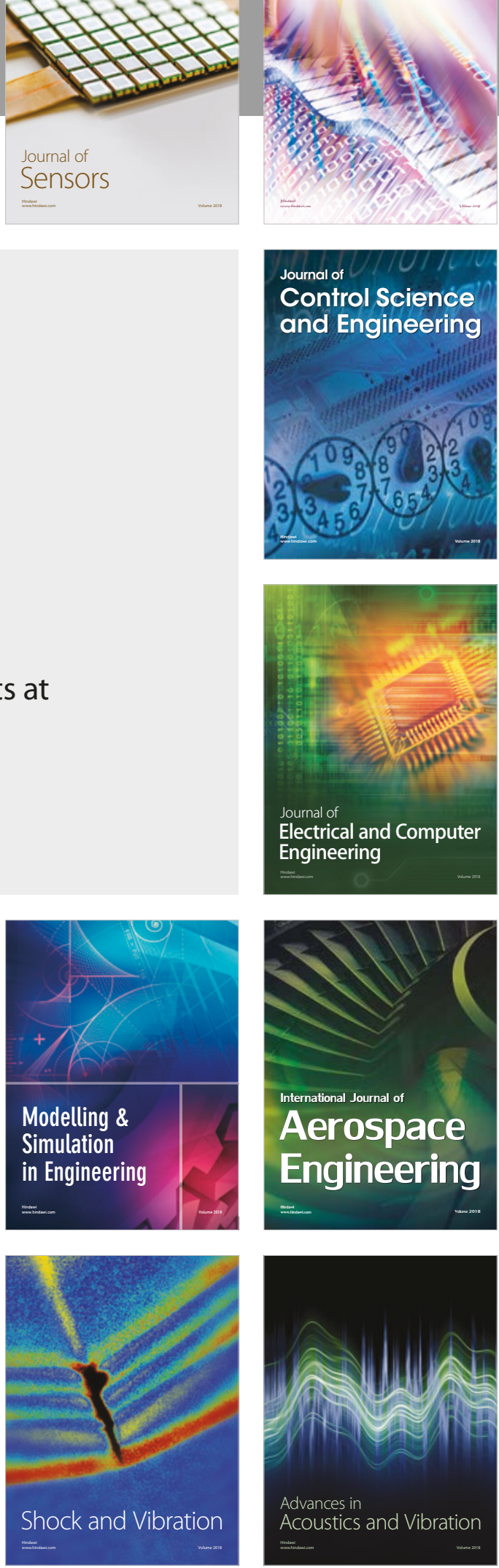